\title{
Improving the wireless communication system at a large Antwerp car factory
}

\author{
Emmanuel Van Lil, Iris De Coster \\ ESAT/TELEMIC \\ KU Leuven \\ Heverlee-Leuven, Belgium \\ Emmanuel.VanLil@ESAT.KULeuven.Be
}

\begin{abstract}
The car factory experienced many communication problems within their factory, due to (new) requirements of the Belgian Federal Communication authorities (BIPT) to limit the height of the antennas to $12 \mathrm{~m}$ above ground level. This paper deal with the challenge to guarantee a $95 \%$ in this factory, with a large amount of moving metallic parts. The solution turned out to be an array of 4 antennas complemented with an auxiliary antenna.
\end{abstract}

Keywords-coverage improvement; large factory scenario; array antenna; auxiliary antenna.

\section{INTRODUCTION}

The requirements of the BIPT implied that the transmit antenna on the factory was only about $2 \mathrm{~m}$ above the (nearly flat) roof (flat with extra buildings above elevators and assembly chains). The BIPT was imposing no requirements on the receiving antenna, a 4 element high gain antenna. A first series of measurements, showed only a $57 \%$ coverage ( $\$ I I)$. Then an antenna array was designed to try to fulfill the requirements (§III). A last series of measruements showed only an improvement to $81 \%$. This required the addition of an additional antenna to obtain the final $95 \%$ coverage ( $(I V)$.

\section{FIRST MEASUREMENTS}

\section{A. Measurement system}

They involved the use of the old transmit antenna, that was nearly similar to the one in use, a HP 8595 spectrum analyzer (serial 3677U00842), a PC and a bilogarythmic antenna. The whole band between 173 and $174 \mathrm{MHz}$ was scanned with a resolution of $2.5 \mathrm{kHz}$ to account for an eventual frequency drift of the transmitter.

\section{B. Measurements}

The purpose was, as can be seen in Fig. 2, not only to measure the values at the desired transmit frequency, but also to correct for eventual neighboring channel interference. This interference can be seen for instance at $173.955 \mathrm{MHz}$. Around every location point inside the factory (about every 3 bays), 20 random points within one wavelength were measured. A simple DOA (Direction Of Arrival) Diagram obtained by rotating the receiving antenna is shown $(5 \mathrm{~dB} / \mathrm{ring})$ in Fig. 1, shows a Rice distribution, but not too different from a Rayleigh distribution.

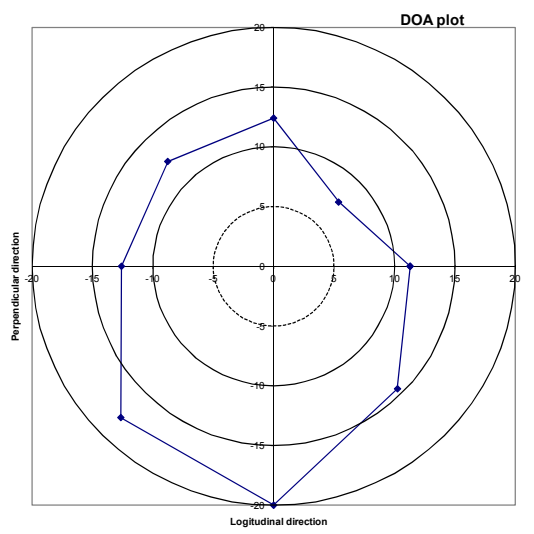

Fig. 1. Sample of mean display for the power in function of frequency at 6 different locations.

The logarithmic mean of those 20 measurements was taken. An example of such a measurement is given in Fig. 2 for the first six positions (all 144 measurements together would give raise to a nearly unreadable picture). The picture shows clearly the frequency of the measurement around $173.89 \mathrm{MHz}$, that was completely cleared of any communication internal to the factory, but the transmission of a CW signal trough the antenna under test, as well as the other of the 5 frequencies being used at the factory. The stability of transmitter and spectrum analyzer was less than $5 \mathrm{kHz}$ over one measurement day. If the levels below $-90 \mathrm{dBm}$ are accounted for as problematic (no or very poor quality, with a Signal to Noise ratio of less than $7 \mathrm{~dB}$ ), the system as it was in 1998 provided a coverage of about $57 \%$ of the factory. This was obviously unacceptable and hence had to be improved. The first measurements were done without auxiliary power supply, so that a lot of time was lost in finding the appropriate power outlets and restarting the computer. Later measurements were performed with a $48 \mathrm{~V}$ DC to $220 \mathrm{~V} \mathrm{AC}$ converter, as well as $412 \mathrm{~V}$ heavy-duty truck batteries provided by the car manufacturer. 


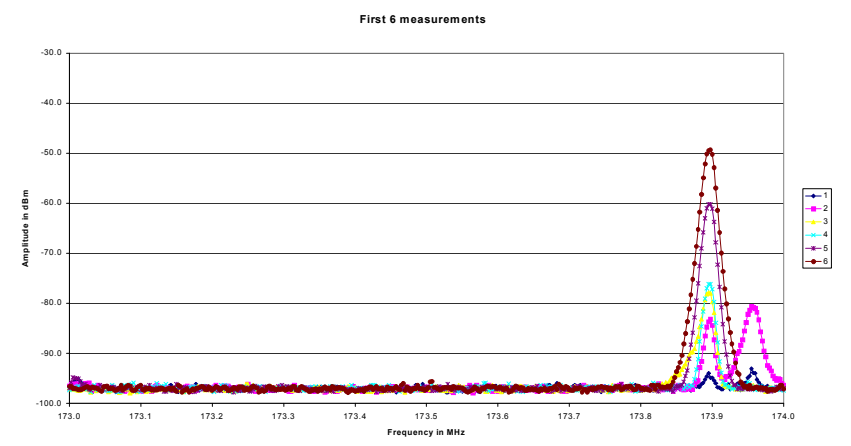

Fig. 2. Sample of mean display for the power in function of frequency at 6 different locations.

As can be seen in Fig. 3, the results were far from good, indicating a coverage of only $57 \%$ of the factory.

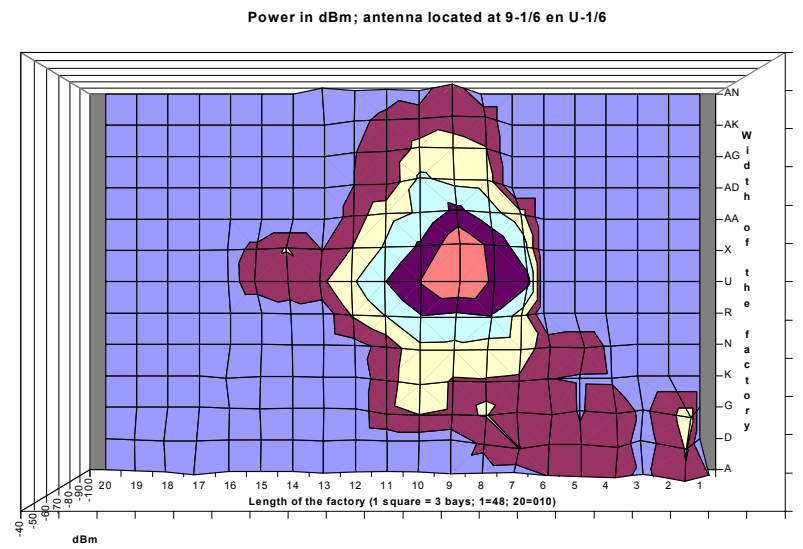

Fig. 3. Measurements before the installation of the improved antenna (coverage $=57 \%$ of the factory).

\section{ANTENNA DESIGN}

To overcome this coverage shortage, several steps were taken. The first was the improvement of the antenna, in collaboration with the Belgian Federal Communication Authorities (BIPT). An antenna was designed that radiated downward and that consisted of 4 linearly polarised elements, that would radiate into the desired band between 173 and 174 MHz. Because the antenna is not visible from the neighborhood of the factory, we obtained the authorisation from the BIPT to raise the level of the antenna, up to $20 \mathrm{~m}$ above the ground (or $10 \mathrm{~m}$ above the lowest roof level).

A $\lambda / 2$ array of 4 vertical dipoles above each other would have solved the problem in one step. The centre of the array would have been at $40 \mathrm{~m}$ above the ground, or $30 \mathrm{~m}$ above the lowest part of the roof, and with phase shifts between each element in such a way that it was radiating downward. This solution however, was rejected by the BIPT. Indeed, they agreed with the correctness of the computations, but did not want to attract imitations. If the phases were not correctly adjusted to radiate downward, the array might produce in that case huge interferences with other communication systems.

An example of the elevation patterns of this antenna, computed with EZNEC [1], that has not been realized, is given in Fig. 4 (also showing the 4 dipoles in purple and their current distributions (green)) and Fig. 5, resp. with and without ground effects. One notices that the levels in the horizontal direction would have been sufficiently low to keep the interferences in the neighborhood minimal.

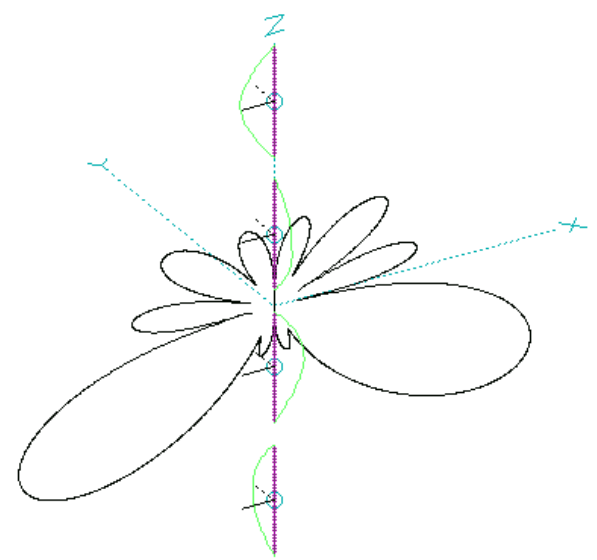

Fig. 4. Elevation pattern of a linear array of 4 vertical dipoles without ground effects.

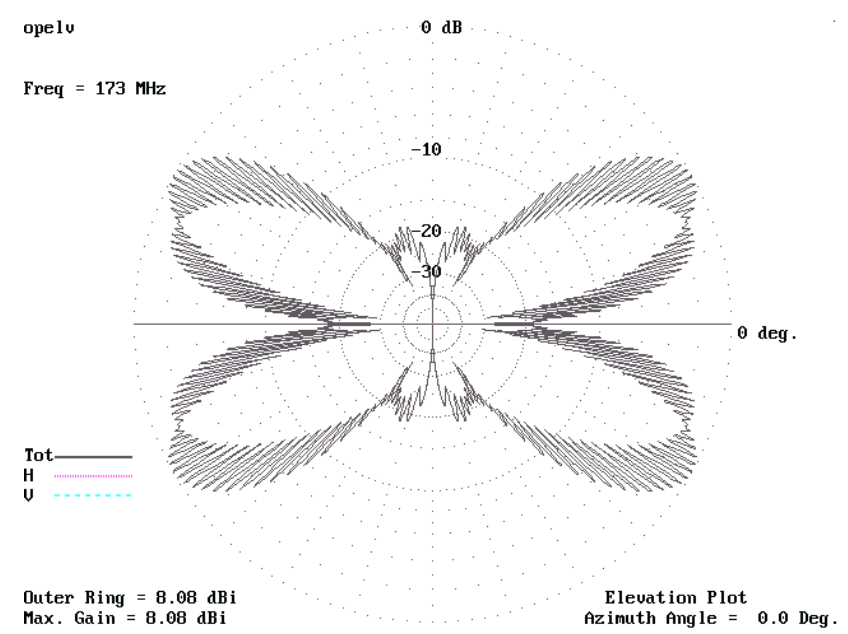

Fig. 5. Elevation pattern of a linear array of 4 vertical dipoles with ground effects.

To achieve a similar gain in the down direction an array was used at a smaller height $(20 \mathrm{~m})$, but with a similar penetration effect through the roof of the factory by using inclined array elements. Those elements were dipoles with a reflecting element arranged as in Fig. 8, that also show the old antenna (just a vertical dipole at a small height above the top of the roof). Radiation patterns in elevation of this array are shown in Fig. 6, and in azimuth in Fig. 7. The azimuthal peaks were directed towards the minima of the signal in the first series of measurements. 


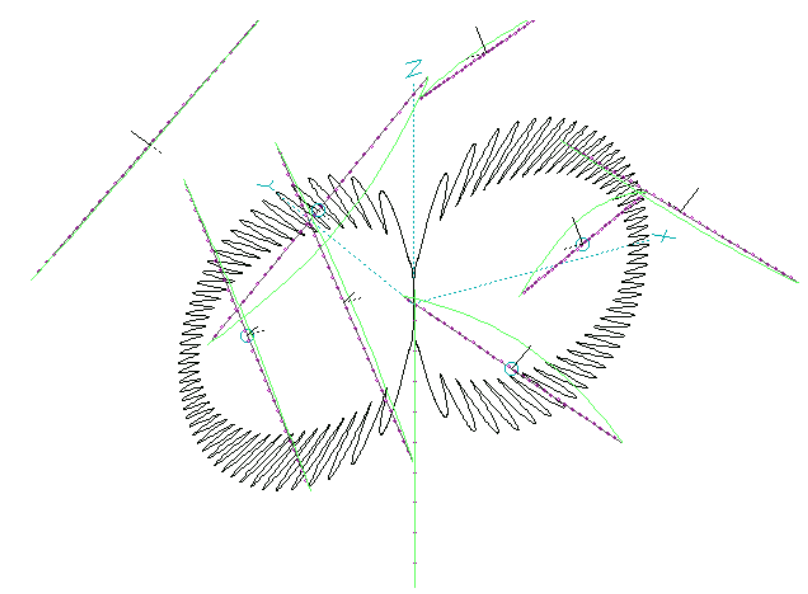

Fig. 6. Elevation pattern of the antenna accepted by the BIPT.

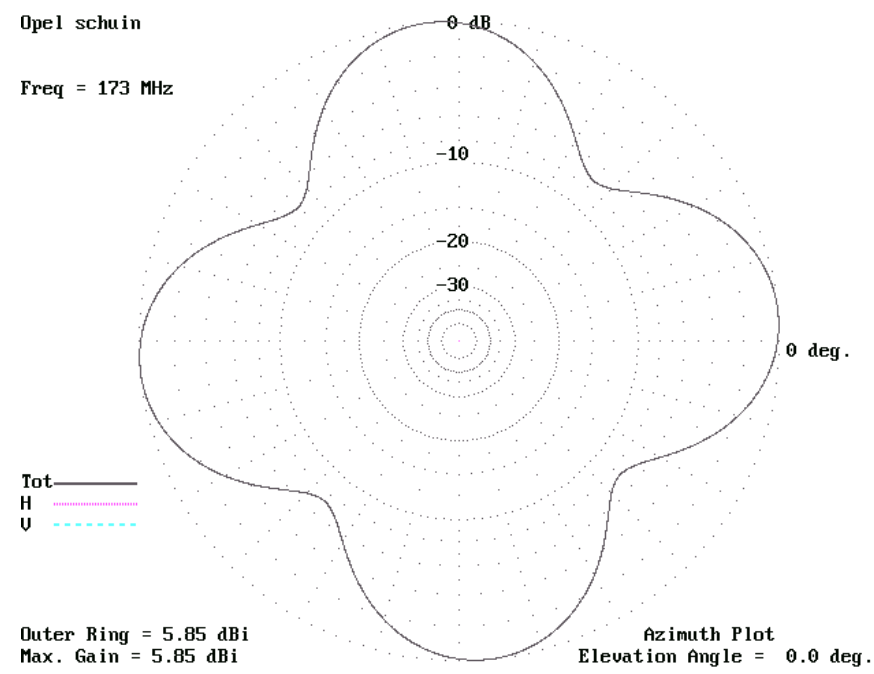

Fig. 7. Azimuthal pattern of the antenna accepted by the BIPT.

To achieve a similar gain in the down direction an array was used at a smaller height $(20 \mathrm{~m})$, but with a similar penetration effect through the roof of the factory by using inclined array elements. Those elements were dipoles with a reflecting element arranged as in Fig. 6, that also show the old antenna (just a vertical dipole at a small height above the top of the roof). Radiation patterns in elevation of this array are shown in Fig. 4, and in azimuth in Fig. 5. The azimuthal peaks were directed towards the minima of the signal in the first series of measurements.
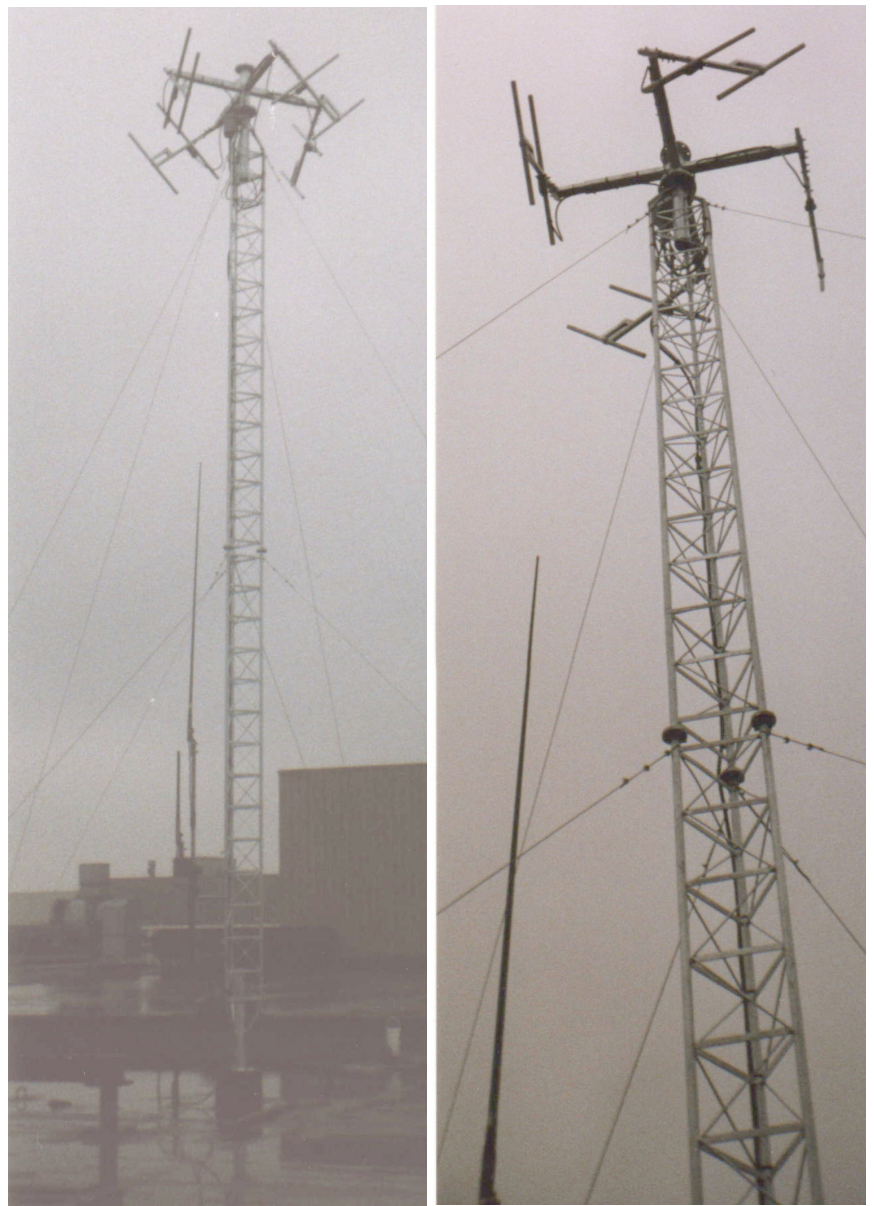

Fig. 8. Views of the antenna on top of the roof after installation.

\section{FINAL MEASUREMENTS}

The measurements, resumed in Fig. 9 show that the coverage was improved (from $57 \%$ to $81 \%$ ) but not yet up to the point that a satisfactory coverage was obtained. Therefor, further improvements had to be made, by installing an auxiliary antenna inside the factory, to cover the problematic areas. The only choice we had, was to couple the transmit power to the auxiliary antenna either via a cable, or via another antenna on the roof of the factory. The auxiliary antenna was placed at location G2 in the centre of the problematic coverage zone. We can deduce that a distance of more than $800 \mathrm{~m}$ is required to gain signal with a third antenna with respect to the cable solution. Because the cable length is approximately $500 \mathrm{~m}$, the cable solution has to be preferred. A $50 \Omega$ discrete element $90^{\circ}$ coupler [2] consisting of 4 capacitances and 4 inductances with a coupling of $-7 \mathrm{~dB}$ in the band between 160 and $180 \mathrm{MHz}$, a matching better than -20 $\mathrm{dB}$ and an insertion loss less than $1.5 \mathrm{~dB}$ in the whole band was designed by TELEMIC and installed. The following measurements were finally satisfying the requirements, because now $96 \%$ of the factory was covered (Fig. 10). 


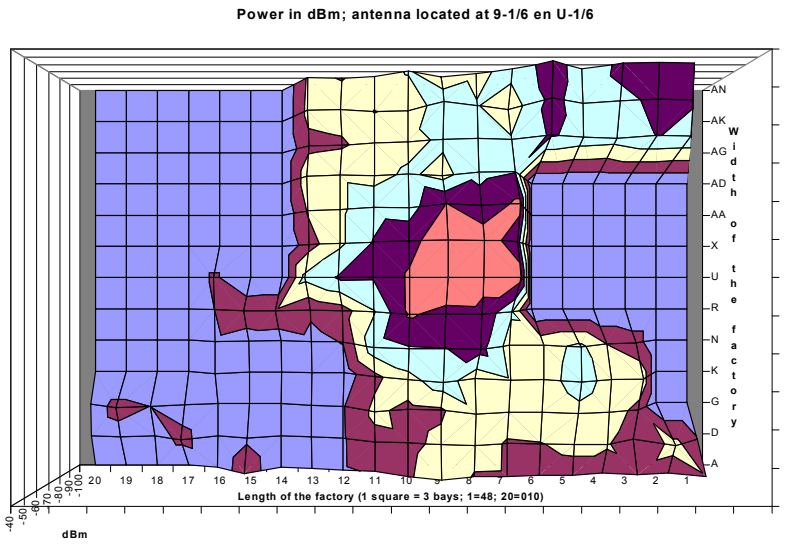

Fig. 9. Measurements after the installation of the improved antenna (coverage $=81 \%$ of the factory).

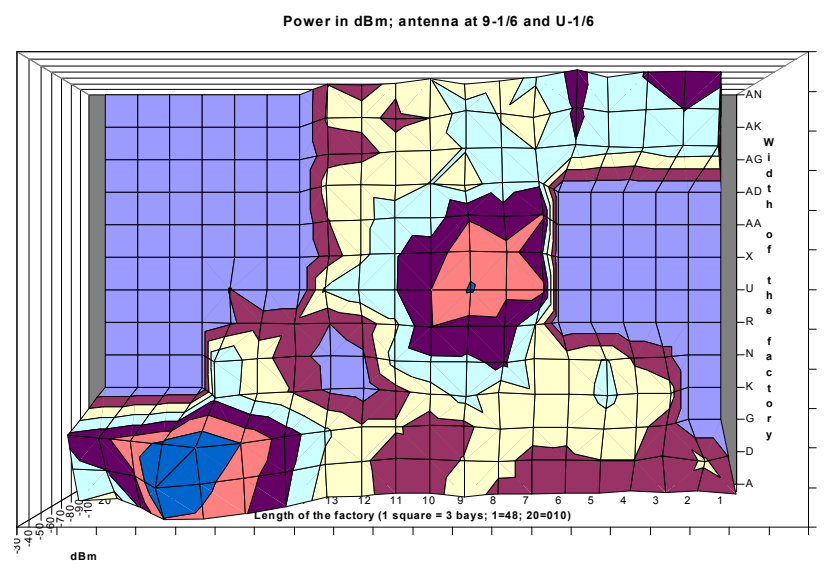

Fig. 10. Measurements after the installation of the auxiliary antenna (coverage $=96 \%$ of the factory).

\section{CONClusions}

To improve the communications to within $95 \%$ of all factory parts, a two step approach was taken leaving only a small part of the factory uncovered. After the installation of the auxiliary antenna, $96 \%$ of the factory surface was covered. The only area that remains below expectation is the area K-N $\& 12-15$. In emergency cases, the persons will have to move about $20 \mathrm{~m}$ away from the centre of this area (L-M \& 13-14) to obtain acceptable communications. It might be possible to improve further the communications in this area by moving the auxiliary antenna closer towards the main antenna.

\section{Acknowledgment}

We are greatly indebted to ing. Hugo Eelen from Opel Antwerpen and irs. Peter Van Huffel and Ivan Vander Beken of the BIPT that made this huge improvement possible within all legal and practical limits of a difficult factory environment.

\section{References}

[1] R. Lewallen, EZNEC, P.O. Box 6658, Beaverton OR 97007, U.S.A.

[2] I Bahl, Lumped Elements for RF and Microwave Circuits, Artech House (June 2003), ISBN-13: 978-1580533096, p.360. 